Annuaire suisse de politique de développement

$14 \mid 1995$

Annuaire Suisse - Tiers Monde 1995

\title{
III. Politique intérieure et extérieure
}

\section{(2) OpenEdition}

1 Journals

Édition électronique

URL : http://journals.openedition.org/aspd/1465

DOI : 10.4000/aspd.1465

ISSN : 1663-9669

Éditeur

Institut de hautes études internationales et du développement

\section{Édition imprimée}

Date de publication : 1 février 1995

Pagination : 87-92

ISSN : 1660-5934

\section{Référence électronique}

«III. Politique intérieure et extérieure », Annuaire suisse de politique de développement [En ligne], 14।

1995, mis en ligne le 04 mai 2013, consulté le 08 septembre 2020. URL : http://

journals.openedition.org/aspd/1465; DOI : https://doi.org/10.4000/aspd.1465

(c) The Graduate Institute I Geneva 


\section{Politique intérieure et extérieure}

\section{Politique d'asile}

Face au nombre toujours croissant de réfugiés, beaucoup de pays cibles des flux migratoires internationaux, dont la Suisse, ont rendu plus restrictive leur pratique de l'asile. La politique d'asile suisse se fonde sur la loi sur l'asile entrée en vigueur en 1981. Depuis lors, cette loi a été révisée à trois reprises. La quatrième révision est annoncé comme étant une révision totale et devrait s'achever en 1996. Les trois révisions précédentes, qui ont toutes restreint la pratique de l'asile, reflètent la réaction de la Suisse face à l'accroissement du nombre de demandes d'asile. A fin 1993, quelque 118000 personnes résidaient en Suisse au titre de la législation sur l'asile, dont 28000 avaient effectivement reçu l'asile. Dans le monde entier, le nombre des réfugiés internationaux et des personnes déplacées dans leur propre pays est estimée à 40 millions. La plupart de ces personnes séjournent dans des pays en développement.

Comme la majorité des autres pays du Nord, la Suisse n'accorde l'asile qu'à très peu de réfugiés; le taux d'acceptation de ces dernières années se situe nettement en-deçà de $10 \%$ (4,5\% en $1992,3 \%$ en 1991), ce qui correspond à la "moyenne européenne". En 1993, ce taux a atteint une moyenne de $20 \%$ (1) en raison du grand nombre de réfugiés provenant de Bosnie-Herzégovine dont la moitié environ se sont vus accorder l'asile. La Suisse octroie toutefois une "autorisation de séjour provisoire" à un grand nombre des requérants qui remplissent les conditions suivantes:

- La décision en matière d'asile n'est pas définitive (recours en suspens), 
- Le renvoi, suite à une décision négative, ne peut être exigé dans certains pays ou dans certaines régions (Sri Lanka),

- Les requérants appartiennent au groupe de réfugiés de la violence (zones de conflit telles que l'ancienne Yougoslavie).

Les réfugiés provenant de pays pour lesquelles la Suisse estime que certaines zones offrent toute la sécurité requise, n'ont pas accès à la procédure d'asile ordinaire. Ces requérants sont renvoyés dans le pays d'origine immédiatement après leur audition, à moins qu'ils ne soient en mesure de prouver qu'ils sont victimes de persécutions. La liste des "pays sûrs" du Conseil fédéral (DFJP et Office fédéral des réfugiés) comprend les pays suivants: Hongrie, Pologne, Bulgarie, Roumanie, Tchéquie, Slovaquie, Albanie, Ghana, Sénégal, Gambie, Inde (état en juin 1994). La Commission de gestion du Conseil national a recommandé à la Confédération de requérir à l'avenir l'avis des oeuvres d'entraide lors de l'analyse de la sécurité des pays et de les inclure dans le processus d'évaluation des pays. Cette solution devrait notamment mettre fin aux controverses qui opposent la Confédération et les oeuvres d'entraide quant à la façon de juger la situation dans un pays.

\section{Demandes d'asile en 1993}

En 1993, 24739 personnes au total ont demandé l'asile en Suisse. Ce nombre reflète à nouveau une augmentation par rapport aux 17960 demandes enregistrées l'année précédente, mais il est nettement inférieur aux statistiques des années précédentes. Comme chaque année depuis 1991, la majeure partie des requérants en 1993 provient de l'ex-Yougoslavie $(27,8 \%$ de Bosnie-Herzégovine et $21,2 \%$ du reste du territoire). D'importants groupes de requérants proviennent de Somalie $(9,3 \%)$, d'Albanie $(8,1 \%)$, du Sri Lanka $(7 \%)$ et de Turquie (4,4\%). (Voir le tableau "Demandes d'asile 1983-1993".) Près de 75\% des requérants sont entrés en Suisse illégalement. La décision d'asile est en principe rendue dans un délai de six mois après le dépôt de la demande. Le taux d'acceptation s'est nettement accru pour les ressortissants de certains pays, notamment pour les réfugiés de Bosnie-Herzégovine $(46,7 \%)$, de Turquie $(26,1 \%)$, tandis qu'il a chuté à $2,1 \%$ pour les personnes provenant du Sri Lanka. Le taux d'acceptation moyen a atteint $20 \%$ en 1993 . Le renvoi des personnes dont la demande d'asile a été définitivement rejetée constitue toujours le problème majeur de la pratique de l'asile. A la fin de 1993, 14000 personnes vivant en Suisse devaient être renvoyées et 14000 autres personnes étaient au bénéfice d'une autorisation de séjour pour motifs humanitaires.

Comme l'indique le vingtième rapport du Système d'observation permanente des migrations de l'OCDE, le mouvement migratoire vers le monde industrialisé s'est ralenti depuis 1992. Selon ce rapport, ce ralentissement s'explique en premier lieu par les conditions plus sévères que la plupart des pays appliquent à l'immigration et à l'asile et, en second lieu seulement, par la dégradation de la situation économique et sociale dans ces pays. L'Allemagne constitue une exception, car elle est submergée par les requérants en prove- 
Tableau $\mathrm{N}^{\circ} 10$

\begin{tabular}{|c|c|c|c|c|c|c|c|c|c|c|c|}
\hline \multicolumn{12}{|c|}{ Demandes d'asiles 1983-1993 } \\
\hline $\begin{array}{l}\text { Pays } \\
\text { d'origine }\end{array}$ & 1983 & 1984 & 1985 & 1986 & 1987 & 1988 & 1989 & 1990 & 1991 & 1992 & 1993 \\
\hline Ethiopie & 153 & 106 & 101 & 150 & 120 & 131 & 204 & 306 & 394 & 94 & 76 \\
\hline Afghanistan & 64 & 33 & 28 & 45 & 80 & 71 & 134 & 234 & 233 & 278 & 144 \\
\hline Albanie & - & - & - & - & - & - & - & 250 & 1038 & 546 & 2009 \\
\hline Algérie & - & - & - & - & . & - & - & - & & - & 751 \\
\hline Angola & 315 & 133 & 371 & 125 & 197 & 322 & 575 & 1134 & 796 & 96 & 542 \\
\hline Bangladesh & 26 & 15 & 34 & 74 & 144 & 195 & 491 & 665 & 593 & 222 & 122 \\
\hline $\begin{array}{c}\text { Bosnie-Her- } \\
\text { zégovie }^{1}\end{array}$ & 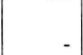 & - & & & . & . & . & - & & - & 6878 \\
\hline Bulgarie & 26 & 21 & 12 & 12 & 8 & 30 & 90 & 681 & 217 & 27 & 25 \\
\hline Chili & 1244 & 487 & 322 & 259 & 291 & 139 & 39 & 11 & 7 & 6 & 1 \\
\hline Tchécos. ${ }^{2}$ & 383 & 214 & 96 & 133 & 96 & 98 & 171 & 21 & 26 & 25 & 12 \\
\hline Ghana & 54 & 58 & 103 & 103 & 101 & 113 & 383 & 450 & 852 & 137 & 66 \\
\hline Inde & 21 & 119 & 114 & 210 & 401 & 730 & 720 & 1828 & 886 & 38 & 21 \\
\hline Irak & 79 & 29 & 12 & 18 & 27 & 34 & 26 & 71 & 160 & 117 & 79 \\
\hline Iran & 112 & 119 & 162 & 951 & 513 & 291 & 211 & 417 & 222 & 140 & 95 \\
\hline Ex-Yougo- & & & & & & & & & & & \\
\hline slavie $^{3}$ & 74 & 102 & 138 & 119 & 131 & 818 & 1365 & 6545 & 14205 & 6262 & 5291 \\
\hline Liban & 19 & 49 & 107 & 144 & 375 & 529 & 2477 & 5533 & 1352 & 450 & 490 \\
\hline Nigeria & 5 & 3 & 2 & 2 & 3 & 8 & 101 & 308 & 515 & 123 & 72 \\
\hline Pakistan & 121 & 364 & 286 & 392 & 581 & 659 & 1027 & 1212 & 1339 & 631 & 410 \\
\hline Pologne & 267 & 319 & 199 & 211 & 160 & 293 & 255 & 549 & 60 & 64 & 16 \\
\hline Roumanie & 245 & 128 & 103 & 105 & 89 & 161 & 431 & 1959 & 2682 & 620 & 113 \\
\hline Somalie & 25 & 37 & 5 & 16 & 22 & 22 & 88 & 181 & 910 & 1077 & 2295 \\
\hline Sri Lanka & 845 & 1236 & 2764 & 593 & 895 & 1516 & 4809 & 4774 & 7349 & 2826 & 1744 \\
\hline Syrie & & 13 & 29 & 138 & 159 & 89 & 115 & 239 & 179 & 146 & 80 \\
\hline Turquie & 1972 & 2639 & 3844 & 4066 & 5817 & 9673 & 9395 & 7262 & 4324 & 1827 & 1205 \\
\hline Hongrie & 261 & 127 & 93 & 70 & 88 & 86 & 16 & & 2 & 3 & 5 \\
\hline Viêt-nam & 88 & 90 & 166 & 174 & 157 & 129 & 168 & 139 & 86 & 69 & 164 \\
\hline Zaïre & 1005 & 756 & 442 & 214 & 191 & 136 & 419 & 758 & 1426 & 677 & 362 \\
\hline Autres & 462 & 228 & 170 & 222 & 267 & 453 & 715 & 1441 & 1731 & 1459 & 1681 \\
\hline Total & 7886 & 7435 & 9703 & 8546 & 10913 & 16726 & 24425 & 36068 & 41584 & 17960 & 24739 \\
\hline
\end{tabular}

1) Les demandes d'asile des requérants des Etats de Bosnie et d'Herzégovie sont dans les banques de données depuis le début de 1993.

2) En 1933: Tchéquie 2; Slovaquie 10.

3) A partir de 1993, reste de l'ex-Yougoslavie sans Croatie (76) ni Slovénie (10) et Macédoine (64)

Source: DFJP/DAR 
nance d'Europe de l'Est. Avec $16,3 \%$ d'étrangers sur la population totale (soit 1,241 million de personnes, dont $10 \%$ séjournant dans le pays sur la base de la législation en matière d'asile), la Suisse se situe en deuxième position parmi les pays de l'OCDE, derrière le Luxembourg ( $28 \%$ de population étrangère). La part des personnes séjournant en Suisse sur la base de la législation en matière d'asile par rapport à la population totale se monte à 1,7\% pour 1993.

\section{Sri Lanka: accord sur le retour de réfugiés}

Ces dernières années, les requérants sri lankais ont bénéficié d'un arrêt de facto des renvois en raison de la guerre civile qui ravage leur pays. Les renvois ont toutefois repris en 1994, après la signature en janvier d'un accord sur le retour de réfugiés passé entre la Suisse et le Sri Lanka. Cet accord devrait garantir aux requérants un retour dans leur patrie "dans la sécurité et la dignité". Les personnes concernées par cette nouvelle pratique en matière d'asile sont au nombre de 12000 environ. Ce sont tous des ressortissants sri lankais dont la demande d'asile a été déposée après le 30 juin 1990 et a été définitivement rejetée. Au total, quelque 18000 Tamouls séjournent en Suisse. Pour ce qui est des renvois, la Confédération applique le principe selon lequel les derniers arrivés sont renvoyés les premiers. L'Office fédéral des réfugiés pose comme hypothèse que la plupart des requérants concernés retournent volontairement dans leur pays. En 1994 et 1995, un total de 700 personnes devraient être renvoyées, tout d'abord en petits groupes.

L'Organisation suisse d'aide aux réfugiés (OSAR, qui est l'organisation faîtière des organismes en faveur des réfugiés) continue de penser que le renvoi des Tamouls au Sri Lanka n'est pas admissible et a exigé de la Confédération que les renvois soient très bien préparés et que les réfugiés renvoyés soient accompagnés et suivis après leur retour. Le Conseil fédéral a refusé de mettre en place la commission d'accompagnement exigée par les oeuvres d'entraide en arguant que le retour au Sri Lanka est certes "non dépourvu de difficultés, mais tout de même possible" (2).

\section{Quatrième révision de la loi sur l'asile}

La quatrième révision de la loi sur l'asile concerne environ deux tiers des dispositions légales; il s'agit avant tout d'adaptations formelles plutôt que de modifications matérielles. Dans le même temps, la révision permettra de reprendre dans la loi les dispositions de l'"Arrêté fédéral urgent sur la procédure d'asile" qui ne peut s'appliquer au-delà de 1995. En lançant la procédure de consultation relative à la révision totale, le Conseil fédéral a souligné que cette révision n'implique pas une réorientation fondamentale de la politique d'asile. Tout comme l'ancienne loi, le nouveau projet pose comme principe que l'asile est accordé uniquement au réfugié qui subit des persécutions individuelles de la part des organes de l'État et qui est en mesure de le prouver. L'inscription dans la loi du statut de réfugié de la violence constitue une innovation. 
Mesures de contrainte en matière de droit des étrangers

Au cours de la session de printemps 1994, le Conseil fédéral a soumis au Parlement une loi visant à créer un droit spécial pour les étrangers sans permis de séjour - ce qui concerne notamment un grand nombre de requérants. II répond ainsi au malaise de la population face aux infractions de certains étrangers/requérants à la loi fédérale sur les stupéfiants et propose d'inscrire des mesures de contrainte dans la législation sur les étrangers. Ces mesures comprennent notamment la détention préparatoire de trois mois et la détention aux fins de refoulement de trois mois pouvant être prolongée à 9 mois. De plus, les étrangers peuvent se voir interdire de pénétrer sur un territoire déterminé ou de le quitter. Les organisations oeuvrant dans le domaine de l'asile et en faveur des réfugiés ont lancé en juillet 1994 un référendum contre la "loi fédérale sur les mesures de contrainte en matière de droit des étrangers" qui avait été adoptée par le Parlement en mars 1994 dans le cadre d'une procédure accélérée. Ce référendum a recueilli plus de 75000 signatures. Les organisations réfé endaires estiment que la loi a été rédigée et acceptée sous le coup de l'émotion et que la procédure accélérée ne s'imposait pas. Elles pensent de plus que la loi laisse une trop grande marge de manoeuvre à la police des étrangers et qu'elle peut engendrer de graves atteintes contre les droits fondamentaux. En instituant cette loi, le Conseil fédéral tenterait de remplacer la politique migratoire et d'intégration - dont les perspectives dépasseraient les frontières de la Suisse - qu'il n'a pas été capable d'élaborer à temps. Le 4 décembre 1994, une majorité de $74 \%$ des votants ont accepté que les nouvelles mesures de contrainte soient intégrées dans la législation sur les étrangers.

\section{Convention sur la suppression de la discrimination raciale}

En 1965, l'Assemblée générale de l'ONU avait adopté la Convention internationale sur la suppression de toute forme de discrimination raciale. La Convention exige des Etats signataires qu'ils luttent activement contre la discrimination raciale et les avantages raciaux.

En 1994, le Conseil fédéral et une majorité parlementaire se sont prononcés pour la ratification de la Convention par la Suisse (avec deux réserves toutefois: limitation de l'accès de ressortissants étrangers au marché du travail suisse en raison du modèle des trois cercles et la garantie de la liberté d'association). Le 25 septembre 1994, le peuple suisse a adopté, à une majorité de $54,7 \%$ des votants, une législation allant dans ce sens. La Suisse est ainsi devenue le 140ème Etat signataire de cette convention universelle.

\section{Autres thèmes en matière d'asile}

- L'initiative déposée en octobre 1993 par l'UDC "contre l'immigration illégale" a officiellement abouti avec 106000 signatures valables (juin 1994). Cette initiative exige le renvoi immédiat de toutes les étrangères et de tous les 
étrangers entrés illégalement en Suisse, tout en tenant compte du principe du non-refoulement (en 1993, ils étaient près de 20'000).

- En février 1994, les partis bourgeois de la droite (les milieux d'extrême droite et les Démocrates suisses) ont à nouveau lancé une initiative concernant les étrangers. Cette initiative populaire "pour une politique d'asile raisonnable" souhaite limiter à $18 \%$ au maximum la part de la population étrangère et mettre fin à "l'incitation financière qui attire les requérants d'asile". En juin 1994, le Conseil fédéral a proposé au Parlement de déclarer cette initiative comme nulle car contraire au droit international public et de rejeter l'initiative de I'UDC sans contre-projet.

\section{Notes}

1. Le taux d'acceptation, qui reflète le nombre d'asiles accordés, tel qu'il est présenté chaque année par l'Office fédéral des réfugiés, mérite quelques réserves si l'on souhaìe s'en servir pour apprécier la situation politique d'un pays. II est certes vrai que le taux d'acceptation des ressortissants d'un pays augmente lorsque la situation politique dans ce pays s'aggrave, comme ce fut le cas pour les Kurdes de Turquie en 1993. Ce taux est toutefois influencé par la réduction de la montagne de cas en suspens. Chaque année en effet, des décisions sont prises concernant des demandes dont certaines ont été déposées des années auparavant.

2. Citation du conseiller fédéral Koller dans la NZZ du 21.4.1994 (Beginn der Rückschiebung nach Sri Lanka - Début des renvois au Sri Lanka): Les Tamouls doivent être traités sur un pied d'égalité avec les autres requérants d'asiles, car la situation dans leur pays est meilleure que celle en Turquie par exemple.

\section{Sources}

Office fédéral des réfugiés, Statistique en matière d'asile 1993, Berne 1994 et la revue Asylon, éditions de 1993 jusqu'en juin 1994

OCDE, Tendances des migrations internationales, 20ème rapport annuel (1993) du Système d'observation permanente des migrations, Paris 1994

ai-Magazin, no 3 / mars 1994 (Sri Lanka: île de la peur / mesures de contrainte en matière de droit des étrangers: l'arbitraire inscrit dans la loi)

BODS/MODS/MADS, Circulaires de 1993 et 1994

Tages-Anzeiger, 23.4.; 11.5.; 2.6.; 3.6.; 24.6.; 5.7.; 26.9.1994

NZZ, 19.4.; 21.4.; 7.6.; 24.6.; 5.7.; 26.9.1994 